\title{
Contribution of inter- and intraband transitions into electron-phonon coupling in metals
}

\author{
Nikita Medvedev ${ }^{1,2, a}$ (D) and Igor $\operatorname{Milov}^{3}$ (D) \\ 1 Institute of Physics, Czech Academy of Sciences, Na Slovance 2, 18221 Prague 8, Czech Republic \\ 2 Institute of Plasma Physics, Czech Academy of Sciences, Za Slovankou 3, 18200 Prague 8, Czech Republic \\ 3 Industrial Focus Group XUV Optics, MESA + Institute for Nanotechnology, University of Twente, Drienerlolaan 5 , \\ 7522 NB Enschede, The Netherlands
}

Received 16 March 2021 / Accepted 14 June 2021 / Published online 23 July 2021

(C) The Author(s) 2021

\begin{abstract}
We recently developed an approach for calculation of the electron-phonon (electron-ion in a more general case) coupling in materials based on tight-binding molecular dynamics simulations. In the present work, we utilize this approach to study partial contributions of inter- and intraband electron scattering events into total electron-phonon coupling in $\mathrm{Al}, \mathrm{Au}$, and $\mathrm{Cu}$ elemental metals and in $\mathrm{AlCu}$ alloy. We demonstrate that the interband scattering plays an important role in the electron-ion energy exchange process in $\mathrm{Al}$ and $\mathrm{AlCu}$, whereas intraband $d-d$ transitions are dominant in $\mathrm{Au}$ and $\mathrm{Cu}$. Moreover, interand intraband transitions exhibit qualitatively different dependencies on the electron temperature. Our findings should be taken into account for the interpretation of experimental results on the electron-phonon coupling parameter.
\end{abstract}

\section{Introduction}

Since the advent of powerful femtosecond lasers, the field of material response to irradiation has been developing fast [1]. It is driven by a wide range of applications in materials surface and bulk processing and nanostructuring for photonics [2], catalysis [3] and biomedicine [4]. Elemental metals and alloys are a class of materials that is widely used in the ultrafast community for its relative simplicity and versatile functionality [5-7]. An ultrafast transfer of the absorbed laser energy from an electronic system of metal to the lattice is a core process that defines the nature and dynamics of irradiated target evolution. Understanding and quantifying such processes is important to keep advancing the field of ultrafast light-matter interaction.

Most often, the response of metals to ultrafast laser pulses is modeled with the two-temperature model

This work benefited from networking activities carried out within the EU funded COST Action CA17126 (TUMIEE) and represents a contribution to it. The authors gratefully acknowledge financial support from the Czech Ministry of Education, Youth and Sports (Grants Nos. LTT17015, EF16_013/0001552, and LM2015083). I. Milov gratefully acknowledges support from the Industrial Focus Group XUV Optics of the MESA+ Institute for Nanotechnology of the University of Twente; the industrial partners ASML, Carl Zeiss SMT GmbH, and Malvern PANalytical, and the Netherlands Organisation for Scientific Research (NWO).

${ }^{a}$ e-mail: nikita.medvedev@fzu.cz (corresponding author)
(TTM) - a set of coupled differential equations for the electronic and atomic/phononic heat conduction and exchange $[8,9]$. The latter is controlled with an electron-phonon coupling parameter, which, in a general case, is a function of many variables defining a material transient state, such as electron and ion temperature, density, etc. [10]. Despite shortcomings of the TTM approach (see, e.g., discussions in Refs. [11,12]), it remains one of the most widely used models in the community.

Further refinements of the model are being developed and applied, resulting in multi-temperature approaches, treating different electronic bands and/or different phonon modes separately, each with its own temperature $[13,14]$, and hence with different energy exchanges among them. Decoupling the contributions into the total coupling from the different electronic bands and interband transitions can help in the further development of advanced models that trace different bands separately, such as, e.g., in Refs. [15-17]. Such models require reliable calculations of various contributions to the coupling parameter.

Here, we use the recently developed method of calculating the electron coupling to the ionic motion [10] and derive contributions of various interband (between different electronic bands) and intraband (within one band) electronic transitions to the total coupling parameter in aluminum, copper, gold, and $\mathrm{AlCu}$ alloy. We focus on a dependence of these partial couplings on the electronic temperature. 


\section{Model}

Electronic coupling to atomic/ionic motion is a process in which an electron transition from one energy level to another occurs, while the energy difference is transferred to or from the atoms. Each atomic displacement induces a change in the Hamiltonian, and correspondingly in its eigenfunctions and eigenstates. These sudden changes from one time step to another trigger electron transfers between the energy levels [18], known as nonadiabatic coupling between atomic displacements and the electronic wave function. In the solid-state community, it is known as the electron-phonon coupling when the atomic motion is harmonic within an ideal crystal lattice.

We use XTANT-3 method described in Ref. [10] to calculate the electron-ion coupling parameter of selected materials. We use the term "electron-ion" instead of a more common "electron-phonon" since our model works beyond the harmonic approximation of the atomic system. (Hence, it is also capable of calculations of the coupling parameter in the disordered matter.) The model is based on tight-binding molecular dynamics simulations to evaluate the evolution of the Hamiltonian, which is dependent on transient positions of all atoms in the simulation box. A solution of the secular equation provides electron wave functions and eigenstates at each molecular dynamics time step, together with the interatomic forces [19]. Knowledge of the transient wave functions allows calculating the matrix elements of electrons coupling to ionic displacements [10]. Using the linear combination of atomic orbitals (LCAO) basis set $\left(c_{i, \alpha}\right)$ within the tight-binding Hamiltonian, a wave function is presented as $\psi_{i}=\sum_{\alpha} c_{i, \alpha} \phi_{\alpha}$, and the electron transition rate between the eigenstates $i$ and $j$ can be written in the following manner [10]:

$$
w_{i j}=\sum_{\alpha, \beta} w_{i j}^{\alpha \beta}=\frac{4 e}{\hbar \delta t^{2}} \sum_{\alpha, \beta}\left|c_{i, \alpha}(t) c_{j, \beta}\left(t_{0}\right) S_{\alpha, \beta}\right|^{2}
$$

where $e$ is the electron charge, $\hbar$ is the Planck's constant, $S_{\alpha, \beta}$ is the tight binding overlap matrix, and the wave functions are calculated at two sequential molecular dynamics time steps $t_{0}$ and $t=t_{0}+\delta t$, where $\delta t$ is the molecular dynamics time step.

The evaluated matrix elements (1) are then used in the Boltzmann collision integral to calculate the energy exchange rate between electrons and ions:

$$
\begin{aligned}
& I_{i j}^{e-a} \\
& =w_{i j} \begin{cases}f\left(E_{j}\right)\left[2-f\left(E_{i}\right)\right]-f\left(E_{i}\right)\left[2-f\left(E_{j}\right)\right] e^{-E_{i j} / T_{a}} & \text { for } i<j \\
f\left(E_{j}\right)\left[2-f\left(E_{i}\right)\right] e^{-E_{i j} / T_{a}}-f\left(E_{i}\right)\left[2-f\left(E_{j}\right)\right] & \text { otherwise }\end{cases}
\end{aligned}
$$

where $f\left(E_{i}\right)$ is the Fermi-Dirac distribution with the defined electronic temperature $T_{\mathrm{e}}, E_{i j}=E_{i}-E_{j}$ is the difference between the energies of the two levels, and $T_{a}$ is the atomic temperature. Knowledge of the
Boltzmann collision integral allows us to evaluate the coupling parameter defined as the energy flow per a volume unit (simulation box volume, $V$ ) and the temperature difference [10]:

$$
G\left(T_{\mathrm{e}}\right)=\frac{1}{\left(T_{\mathrm{e}}-T_{a}\right) V} \sum_{i, j} E_{i} I_{i j}^{e-a}
$$

For each material considered, the molecular dynamics simulations are performed ten times with varied initial conditions (slightly different durations of atomic relaxation prior to the productive simulation run, random atomic velocities corresponding to Maxwellian distribution at the room temperature) and parameters of the electronic temperature increase (increase rate and the maximal aimed temperature), to deliver reliable averaged electron-ion energy exchange rates. In Ref. [10] it was demonstrated that this method of calculation provides a good agreement with available experimental data on the electron-phonon coupling in metals at elevated electronic temperatures. The convergence for gamma-point calculations in the Brillouin zone with respect to the number of atoms in the simulation box and to the time step was also thoroughly studied in Ref. [10] - the same converged parameters are used here.

In the present work, we calculate the coupling parameter decomposed into corresponding contributions from the atomic orbitals that form the LCAO basis set $\left(w_{i j}^{\alpha \beta}\right)$. The employed procedure is analogous to the standard definition of partial density of states (pDOS), or Mulliken atomic charges. It assigns contributions of atomic orbitals into each electronic shell/band, thus dividing the total coupling into partial coupling contributions from electron transitions between the corresponding bands. The interband contributions in our calculations are the sum of direct and inverse transitions: $(\alpha-\beta)$ is a sum of both, $\alpha \rightarrow \beta$ (terms with $\left.c_{i, \alpha}(t) c_{j, \beta}\left(t_{0}\right)\right)$ and $\beta \rightarrow \alpha$ (terms with $\left.c_{i, \beta}(t) c_{j, \alpha}\left(t_{0}\right)\right)$ transitions. For example, the partial coupling between the states $s-p$ is a sum of $s \rightarrow p$ and $p \rightarrow s$, etc.

The following examples are considered here: elemental metals $\mathrm{Al}, \mathrm{Au}, \mathrm{Cu}$, and $\mathrm{AlCu}$ alloy. For the elemental metals we used the same TB parameterization as in our previous work [10] (NRL TB parameterization [20]), whereas for AlCu alloy we used DFTB matsci-03 parameterization [21]. It is also, like NRL, based on $s p^{3} d^{5}$ LCAO basis set. We assumed AlCu to be in a simple cubic lattice structure and used a supercell with 250 atoms. We used a 1 fs time step in the molecular dynamics part of our model. The NRL and DFTB parameterizations use relatively large basis sets, and relatively long cut-off distances, accounting for the interaction of many neighboring atoms (not only the nearest neighbors). They also have been cross-checked to describe solids and molecules with reasonable accuracy [20,21]. Our calculations of the coupling parameter for $\mathrm{Al}$ and Au targets were cross-checked in Ref. [10] (and shown in Figs. 1, 2, and 3) at elevated electronic temperatures. As for the $\mathrm{AlCu}$ coupling parameter (Fig. 4), our calcu- 


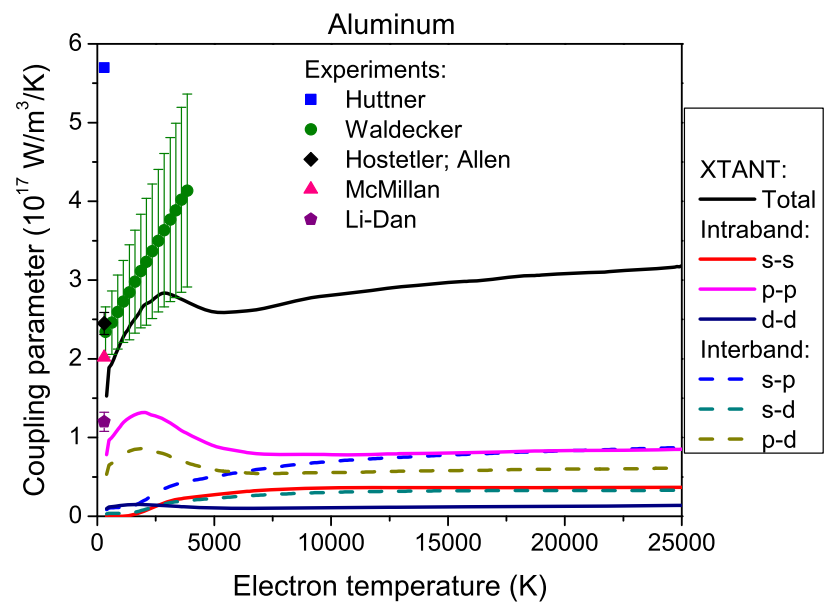

Fig. 1 XTANT-3 calculated total and partial electron-ion coupling in aluminum. Experimental data shown for comparison include those of Hüttner and Rohr [11], Waldecker et al. [13], Hostetler et al. [22], Allen and Cohen [23], and Li-Dan et al. [24]

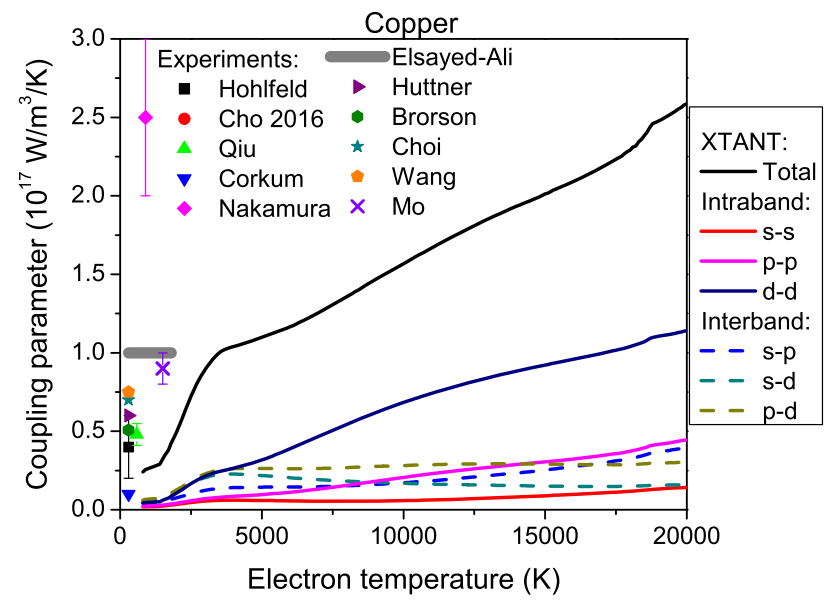

Fig. 2 XTANT-3 calculated total and partial electron-ion coupling in copper. Experimental data shown for comparison include those of Hohlfeld et al. [25], Qiu and Tien [26], Nakamura et al. [27], Corkum et al. [28], Elsayed-Ali et al. [29], Hüttner and Rohr [11], Brorson et al. [30], Mo et al. [31], Choi et al. [32], and Cho et al. [33]

lations must be validated in the future against experimental data.

\section{Results}

Figure 1 shows the coupling parameter in aluminum. The total coupling first increases with the increase in the electron temperatures and then has a slight dip followed by a slow increase further. As was discussed in Ref. [10], the peaks in the coupling seem to correspond to the peaks in the electronic DOS near the Fermi energy. When a lot of electrons (and a lot of unoccupied electronic states) are available, it allows for many elec-

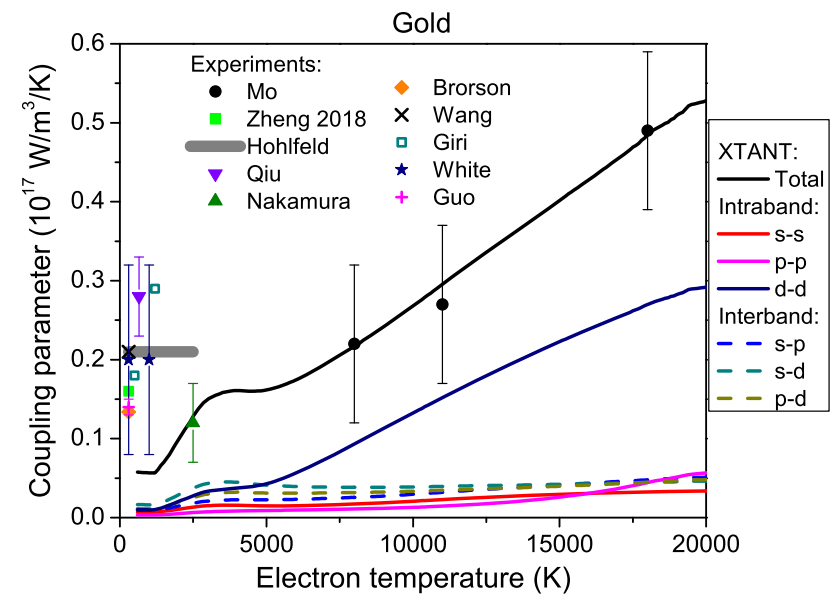

Fig. 3 XTANT-3 calculated total and partial electron-ion coupling in gold. Experimental data shown for comparison include those of Mo et al. [34], Hohlfeld et al. [25], Qiu and Tien [26], Nakamura et al. [27], Brorson et al. [30], Zheng et al. [35], Giri et al. [36], White et al. [37], and Guo and Xu [38]

tron transitions which result in energy exchange with the atoms. With further increase in the electronic temperature, the Fermi-Dirac distribution smears out, not concentrating around the peaks, and averaging out with small DOS, which may reduce the overall energy transfer to the atoms.

The partial couplings attributed to various electronic transitions have qualitatively different dependencies on the electronic temperature. At low electronic temperatures, the main contribution to the total coupling is from the transitions between $p-p$ and $p-d$ shells, whereas with the increase in the electronic temperature, contributions from interband $s-p$ and $s-d$ transitions, as well as from the intraband $s-s$ transition, start to play a larger role. The $d-d$ transitions make only a minor contribution to the total coupling parameter.

In contrast to aluminum, the main contribution to the increasing total coupling parameter in copper (Fig. 2) and gold (Fig. 3) comes from $d-d$ transitions. Partial couplings attributed to other transitions exhibit a weak dependence on the electronic temperature and have smaller contributions to the total coupling parameter. In gold, $d$-band containing 10 electrons is located at $\sim 2 \mathrm{eV}$ below the Fermi level [39]; thus, at low electronic temperatures this band does not participate in electron-ion coupling. With the increase in the electron temperature, more and more electrons from the $d$-band are involved in the coupling, which increases $d-d$ partial coupling, making it dominant at electronic temperatures above $\sim 5000 \mathrm{~K}$. A similar concept for copper was discussed in Ref. [40]. In contrast, the partial DOS in aluminum does not have such a strong localization [39] and all the bands contribute to the electron-ion coupling, especially at elevated electronic temperatures.

In $\mathrm{AlCu}$ alloy, we decomposed the contributions into bands formed by shells of different atoms (Al and $\mathrm{Cu}$, and interatomic contributions) and into transitions 


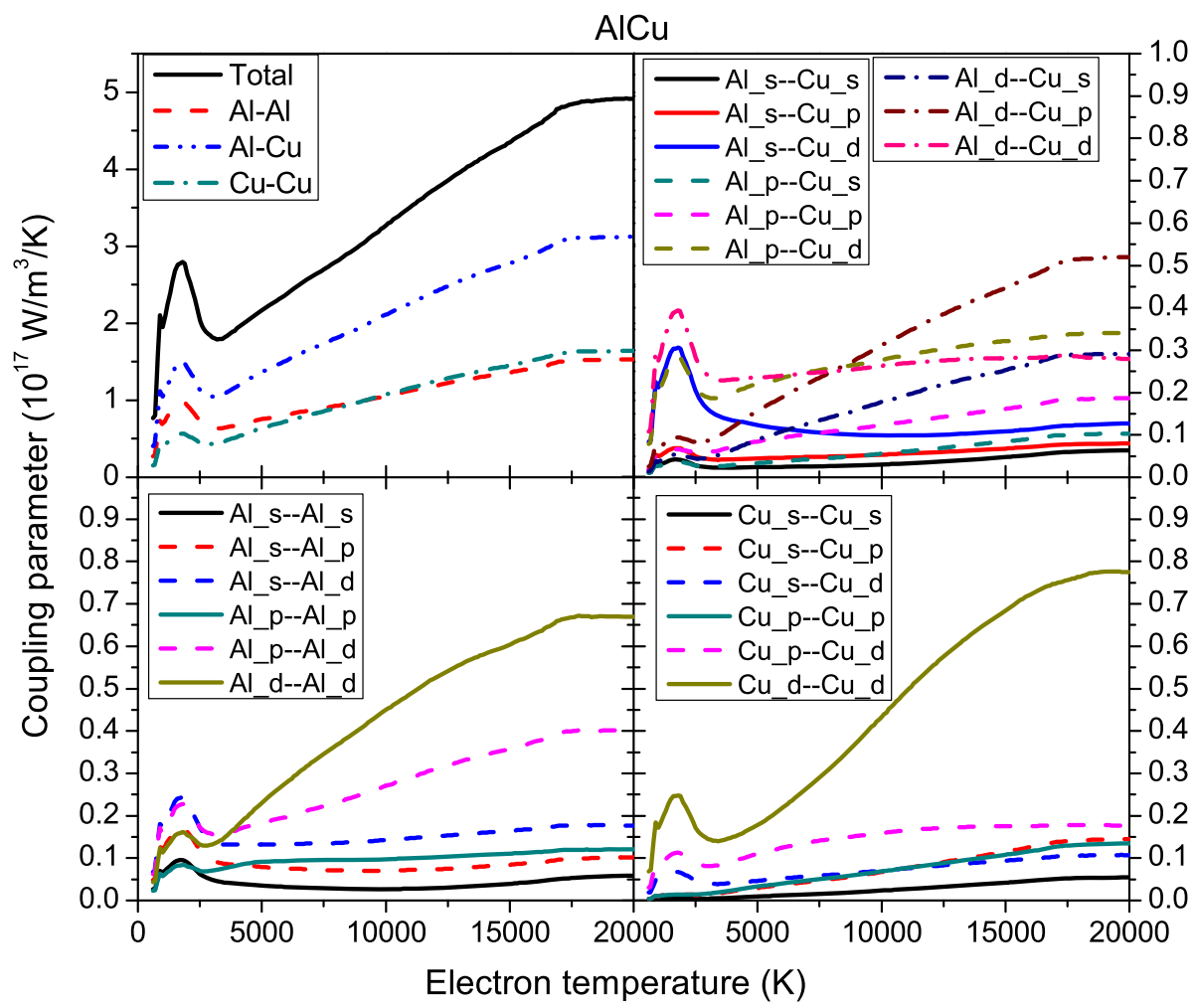

Fig. 4 XTANT-3 calculated electron-ion coupling in AlCu alloy. Top left panel: total coupling and coupling decomposed into transitions between bands formed by $\mathrm{Al}$ and $\mathrm{Cu}$ atoms. Top right panel: interatomic interband contributions into coupling. Bottom left panel: $\mathrm{Al}$ inter- and intraband contributions. Bottom right panel: $\mathrm{Cu}$ inter- and intraband contributions

between various shells in those atoms, which is shown in different panels in Fig. 4. There, the dominant contributions are from interatomic transitions between the bands formed by $\mathrm{Al}$ and $\mathrm{Cu}$ atoms (top left panel). Contributions of interband transitions are dominant, however with noticeable intraband transitions within $d$ bands of $\mathrm{Al}$ and of $\mathrm{Cu}$ (other panels in Fig. 4). Note again that at different electronic temperatures, different bands have dominant contributions. Scattering within $d$ bands of $\mathrm{Al}$ and of $\mathrm{Cu}$ atoms in $\mathrm{AlCu}$ alloy, as well as interband transitions that involve $d$ bands, has a dominant effect. At low electronic temperatures, the main peak is formed by the transitions between the $d$ band of $\mathrm{Cu}$ and other bands of $\mathrm{Al}$. With the increase in the electronic temperature, the scattering between $d$-band electrons of $\mathrm{Al}$ and other bands increases (as well as $d-d \mathrm{Cu}$ transitions).

\section{Discussion}

In all calculated materials, most prominently in aluminum and $\mathrm{AlCu}$ alloy, interband electron transitions play a significant role in electron-ion energy exchange. Such an observation indicates that models accounting only for intraband coupling, even if band-resolved (such as, e.g., in Refs. [17,41] where $s$ or $s p$ and $d$ bands were considered to be coupling to phonons separately), may overlook important contributions. Even in gold and copper, albeit the $d-d$ contribution is dominant at high electronic temperatures, it is still necessary to account for interband transitions. The importance of interband transitions for electron-phonon coupling was also discussed in a series of works by Hopkins et al. [1416,40], albeit within the Eliashberg approximation [42] which overestimates the coupling parameter at nonzero electronic temperatures [10].

The presented calculations assumed thermal equilibrium within the electronic system - the electron distribution function always follows the Fermi-Dirac distribution, with the same temperature and chemical potential in all electronic bands. In experiments, it may not always be the case. When electrons are predominantly excited from one band into another one [17], for example, due to particular tuning of the optical photon energy, particular electron transitions may be enhanced or inhibited. For example, one may expect that excitation of electrons only from the $s$-band of gold by photon energy below $2 \mathrm{eV}$ (without reaching $d$-band electrons) may suppress the electron coupling due to a lack of $d-d$ transitions, which are the dominant contribution into the increase in the coupling with the rise of the electronic temperature, see Fig. 3. In such a nonequilibrium case, with only $s$ but not $d$-electrons excited, the contribution into the coupling will be smaller than 
in the case when $d$-electrons are contributing (such as in the equilibrium case). It was suggested that this kind of nonequilibrium within the electronic system may last for significantly long times in gold [17]. It was demonstrated experimentally for nickel that irradiation with photon energies exciting $d$-band induces significantly different electron-phonon coupling compared to irradiation with photon energies insufficient to excite $d$-band electrons [15].

This fact has large implications for experiments with ultrafast optical laser irradiation. Excitation of electrons into a nonequilibrium state may reduce the observable coupling parameter with respect to the situation when electrons are in thermal equilibrium (which corresponds to the total coupling in Figs. 2 and 3). This may be the reason why experimental works on ultrafast irradiation of gold often report constant or nearly constant coupling parameter at different electronic temperatures [34, 43, 44].

\section{Conclusions}

We calculated partial electron-ion coupling parameters in $\mathrm{Al}, \mathrm{Cu}, \mathrm{Au}$ and $\mathrm{AlCu}$ alloy. Contributions of inter- and intraband electronic transitions are presented, which may be useful for models beyond the two temperature approximation. We found that the intraband contribution in all materials, most pronouncedly in copper and gold, behaves qualitatively different from interband contributions with the increase in the electronic temperature. In particular, $d-d$ electron transitions in gold and copper play the dominant role at electron temperature above $\sim 5000 \mathrm{~K}$. This fact has implications for the interpretation of ultrafast lightmatter interaction experiments, in which electronic system may be out of equilibrium due to a particular choice of incident photon energy.

Acknowledgements The authors thank B. Rethfeld, P. Ndione, and $\mathrm{A}$. $\mathrm{Ng}$ for motivating discussions. Computational resources were supplied by the project "e-Infrastruktura CZ" (e-INFRA LM2018140) provided within the program Projects of Large Research, Development and Innovations Infrastructures.

Data Availability Statement This manuscript has no associated data or the data will not be deposited. [Authors' comment: Data used for the plots may be obtained from the authors upon a request.]

Open Access This article is licensed under a Creative Commons Attribution 4.0 International License, which permits use, sharing, adaptation, distribution and reproduction in any medium or format, as long as you give appropriate credit to the original author(s) and the source, provide a link to the Creative Commons licence, and indicate if changes were made. The images or other third party material in this article are included in the article's Creative Commons licence, unless indicated otherwise in a credit line to the material. If material is not included in the article's Creative Commons licence and your intended use is not permitted by statutory regulation or exceeds the permitted use, you will need to obtain permission directly from the copyright holder. To view a copy of this licence, visit http://creativecomm ons.org/licenses/by/4.0/.

\section{References}

1. W.E. Lamb, W.P. Schleich, M.O. Scully, C.H. Townes, Rev. Mod. Phys. 71(SUPPL. 2), S263 (1999). https:// doi.org/10.1103/revmodphys.71.s263

2. A. Stone, H. Jain, V. Dierolf, M. Sakakura, Y. Shimotsuma, K. Miura, K. Hirao, J. Lapointe, R. Kashyap, Sci. Rep. 5(1), 1 (2015). https://doi.org/10.1038/srep10391

3. P. Nuernberger, D. Wolpert, H. Weiss, G. Gerber, Phys. Chem. Chem. Phys. 14(3), 1185 (2012). https://doi. org/10.1039/C1CP21827A

4. V. Melissinaki, A.A. Gill, I. Ortega, M. Vamvakaki, A. Ranella, J.W. Haycock, C. Fotakis, M. Farsari, F. Claeyssens, Biofabrication 3(4), 45005 (2011). https:// doi.org/10.1088/1758-5082/3/4/045005

5. A.Y. Vorobyev, C. Guo, Opt. Express 14(6), 2164 (2006). https://doi.org/10.1364/OE.14.002164

6. P. Bizi-bandoki, S. Valette, E. Audouard, S. Benayoun, Appl. Surf. Sci. 273, 399 (2013). https://doi.org/10. 1016/j.apsusc.2013.02.054

7. R. Jagdheesh, B. Pathiraj, E. Karatay, G.R.B.E. Römer, A.J. in't Veld, Langmuir 27(13), 8464 (2011). https:// doi.org/10.1021/la2011088

8. M.I. Kaganov, I.M. Lifshits, L.V. Tanatarov, J. Exp. Theor. Phys. 31, 232 (1956)

9. S.I. Anisimov, B.L. Kapeliovich, T.L. Perel-man, J. Exp. Theor. Phys. 39(2), 375 (1974)

10. N. Medvedev, I. Milov, Phys. Rev. B 102(6), 064302 (2020). https://doi.org/10.1103/PhysRevB.102.064302

11. B. Hüttner, G. Rohr, Appl. Surf. Sci. 103(3), 269 (1996). https://doi.org/10.1016/0169-4332(96)00523-5

12. B. Hüttner, (2017), pp. 341-361. https://doi.org/10. 1007/978-3-319-56711-2_11

13. L. Waldecker, R. Bertoni, R. Ernstorfer, J. Vorberger, Phys. Rev. X 6(2), 021003 (2016). https://doi.org/10. 1103/PhysRevX.6.021003

14. P.E. Hopkins, J. Heat Transf. 132, 12 (2010). https:// doi.org/10.1115/1.4002295

15. P.E. Hopkins, J.M. Klopf, P.M. Norris, Appl. Opt. 46(11), 2076 (2007). https://doi.org/10.1364/AO.46. 002076

16. P.E. Hopkins, D.A. Stewart, J. Appl. Phys. 106, 5 (2009). https://doi.org/10.1063/1.3211310

17. P.D. Ndione, S.T. Weber, B. Rethfeld, D.O. Gericke, Contrib. Plasma Phys. 59(4-5), e201800186 (2019). https://doi.org/10.1002/ctpp.201800186

18. J.C. Tully, J. Chem. Phys. 93(2), 1061 (1990). https:// doi.org/10.1063/1.459170

19. N. Medvedev, V. Tkachenko, V. Lipp, Z. Li, B. Ziaja, 4open 1, 3 (2018). https://doi.org/10.1051/ fopen/2018003

20. D.A. Papaconstantopoulos, M.J. Mehl, J. Phys. Condens. Matter 15(10), R413 (2003). https://doi.org/10. $1088 / 0953-8984 / 15 / 10 / 201$ 
21. J. Frenzel, A.F. Oliveira, N. Jardillier, T. Heine, G. Seifert, Semi-Relativistic, Self-Consistent Charge Slater-Koster Tables for Density-Functional Based Tight-Binding (DFTB) for Materials Science Simulations. Technical Report, TU-Dresden, Dresden (2009). http://www.dftb.org/parameters/download/matsci/ matsci-0-3-cc/

22. J.L. Hostetler, A.N. Smith, D.M. Czajkowsky, P.M. Norris, Appl. Opt. 38(16), 3614 (1999). https://doi.org/10. 1364/AO.38.003614

23. P.B. Allen, M.L. Cohen, Phys. Rev. 187(2), 525 (1969). https://doi.org/10.1103/PhysRev.187.525

24. Z. Li-Dan, S. Fang-Yuan, Z. Jie, T. Da-Wei, Acta Phys. Sin. 61(13), 134402 (2012). https://doi.org/10.7498/ aps.61.134402

25. J. Hohlfeld, J.G. Müller, S.S. Wellershoff, E. Matthias, Appl. Phys. B Lasers Opt. 64(3), 387 (1997). https:// doi.org/10.1007/s003400050189

26. T.Q. Qiu, C.L. Tien, J. Heat Transf. 115(4), 835 (1993). https://doi.org/10.1115/1.2911377

27. A. Nakamura, T. Shimojima, M. Nakano, Y. Iwasa, K. Ishizaka, Struct. Dyn. 3, 6 (2016). https://doi.org/10. 1063/1.4971210

28. P.B. Corkum, F. Brunel, N.K. Sherman, T. SrinivasanRao, Phys. Rev. Lett. 61(25), 2886 (1988). https://doi. org/10.1103/PhysRevLett.61.2886

29. H.E. Elsayed-Ali, T.B. Norris, M.A. Pessot, G.A. Mourou, Phys. Rev. Lett. 58(12), 1212 (1987). https:// doi.org/10.1103/PhysRevLett.58.1212

30. S.D. Brorson, A. Kazeroonian, J.S. Moodera, D.W. Face, T.K. Cheng, E.P. Ippen, M.S. Dresselhaus, G. Dresselhaus, Phys. Rev. Lett. 64(18), 2172 (1990). https://doi.org/10.1103/PhysRevLett.64.2172

31. M.Z. Mo, V. Becker, B.K. Ofori-Okai, X. Shen, Z. Chen, B. Witte, R. Redmer, R.K. Li, M. Dunning, S.P. Weathersby, X.J. Wang, S.H. Glenzer, Rev. Sci. Instrum. 89(10), 10 (2018). https://doi.org/10.1063/1.5035368

32. G.M. Choi, C.H. Moon, B.C. Min, K.J. Lee, D.G. Cahill, Nat. Phys. 11(7), 576 (2015). https://doi.org/10.1038/ nphys 3355

33. B.I. Cho, T. Ogitsu, K. Engelhorn, A.A. Correa, Y. Ping, J.W. Lee, L.J. Bae, D. Prendergast, R.W. Falcone, P.A. Heimann, J. Daligault, S. Gupta, S.H. Glenzer, J.W. Chan, T. Huser, S. Risbud, R.W. Lee, A. Ng, T. Ao, F. Perrot, M.W.C. Dharma-wardana, M.E. Foord, T. Ao, Y. Ping, G.M. Dyer, R. Ernstorfer, A. Mančić, B.I. Cho, T.G. White, Z. Chen, M.W.C. Dharma-wardana, F. Perrot, J. Vorberger, D.O. Gericke, T. Bornath, M. Schlanges, U. Reimann, C. Toepffer, A. Ng, P. Celliers, G. Xu, A. Forsman, D. Riley, Z. Lin, L.V. Zhigilei, V. Celli, B.I. Cho, S. Johnson, J. Hohlfeld, S. Wellershoff, J. Güdde, U. Conrad, H. Elsayed-Ali, T. Norris, M. Pessot, W.L. McMillan, G. Grimvall, E. Wohlfarth, L.B. Fletcher, M.G. Gorman, J. Gaudin, F. Dorchies, G. Paolo, D. Prendergast, G. Galli, Sci. Rep. 6, 18843 (2016). https://doi.org/10. 1038/srep18843
34. M.Z. Mo, Z. Chen, R.K. Li, M. Dunning, B.B.L. Witte, J.K. Baldwin, L.B. Fletcher, J.B. Kim, A. Ng, R. Redmer, A.H. Reid, P. Shekhar, X.Z. Shen, M. Shen, K. Sokolowski-Tinten, Y.Y. Tsui, Y.Q. Wang, Q. Zheng, X.J. Wang, S.H. Glenzer, Science 360(6396), 1451 (2018). https://doi.org/10.1126/science.aar2058

35. Q. Zheng, X. Shen, K. Sokolowski-Tinten, R.K. Li, Z. Chen, M.Z. Mo, Z.L. Wang, S.P. Weathersby, J. Yang, M.W. Chen, X.J. Wang, J. Phys. Chem. C 122(28), 16368 (2018). https://doi.org/10.1021/acs.jpcc.8b03299

36. A. Giri, P.E. Hopkins, J. Appl. Phys. 118(21), 215101 (2015). https://doi.org/10.1063/1.4936606

37. T.G. White, P. Mabey, D.O. Gericke, N.J. Hartley, H.W. Doyle, D. McGonegle, D.S. Rackstraw, A. Higginbotham, G. Gregori, Phys. Rev. B 90(1), 14305 (2014). https://doi.org/10.1103/PhysRevB.90.014305

38. L. Guo, X. Xu, J. Heat Transf. 136, 12 (2014). https:// doi.org/10.1115/1.4028543

39. D.A. Papaconstantopoulos, Handbook of the Band Structure of Elemental Solids (Springer, Boston, 2015). https://doi.org/10.1007/978-1-4419-8264-3

40. P.E. Hopkins, J.C. Duda, R.N. Salaway, J.L. Smoyer, P.M. Norris, Nanoscale Microscale Thermophys. Eng. 12(4), 320 (2008). https://doi.org/10.1080/ 15567260802591985

41. Y.V. Petrov, N.A. Inogamov, K.P. Migdal, JETP Lett. 97(1), 20 (2013). https://doi.org/10.1134/ S0021364013010098

42. Z. Lin, L. Zhigilei, V. Celli, Phys. Rev. B 77(7), 075133 (2008). https://doi.org/10.1103/PhysRevB.77.075133

43. A. Ng, Int. J. Quantum Chem. 112(1), 150 (2012). https://doi.org/10.1002/qua.23197

44. Z. Chen, B. Holst, S.E. Kirkwood, V. Sametoglu, M. Reid, Y.Y. Tsui, V. Recoules, A. Ng, Phys. Rev. Lett. 110(13), 135001 (2013). https://doi.org/10.1103/ PhysRevLett.110.135001 